\title{
Gauss Type Quadrature Rules for Cauchy Principal Value Integrals
}

\author{
By David Elliott and D. F. Paget
}

\begin{abstract}
Two quadrature rules for the approximate evaluation of Cauchy principal value integrals, with nodes at the zeros of appropriate orthogonal polynomials, are discussed. An expression for the truncation error, in terms of higher order derivatives, is given for each rule. In addition, two theorems, containing sufficient conditions for the convergence of the sequence of quadrature rules to the integral, are proved.
\end{abstract}

1. Introduction. For $\lambda \in(a, b)$, let $I(f ; \lambda)$ denote the Cauchy principal value integral

$$
I(f ; \lambda)=f_{a}^{b} \frac{w(x) f(x)}{x-\lambda} d x=\lim _{\epsilon \rightarrow 0}\left(\int_{a}^{\lambda-\epsilon}+\int_{\lambda+\epsilon}^{b}\right) \frac{w(x) f(x)}{x-\lambda} d x,
$$

where the interval $(a, b)$ may be finite or infinite. The weight function $w$ is nonnegative on $(a, b)$ with $\int_{a}^{b} w(x) d x>0$, and we shall assume that $\int_{a}^{b} w(x) x^{n} d x$ exists for $n=0,1,2, \ldots$. It is well known that for such $w$, there exists a sequence of orthogonal polynomials $\left\{p_{n}\right\}$ such that $p_{n}(x)=k_{n} x^{n}+\cdots$ with $k_{n}>0$ for $n=0,1,2, \ldots$, so that $p_{n}$ is of exact degree $n$; and, furthermore,

$$
\int_{a}^{b} w(x) p_{n}(x) p_{m}(x) d x=h_{n} \delta_{m, n}
$$

It also follows that the zeros $x_{i, n}, i=1(1) n$ of $p_{n}$ are real, simple and lie in $(a, b)$. In order to obtain an approximate value of $I(f ; \lambda)$ we shall approximate to it by quadrature sums $Q_{n}$ which use the values of the function $f$ at the points $x_{i, n}, i=1(1) n$. Such quadrature rules are said to be of Gauss type (see [5]).

Two closely related Gauss type methods for the approximate evaluation of $I(f ; \lambda)$ were published in 1972 by Paget and Elliott [14] and Hunter [9]. For a given $n$, Paget and Elliott approximated to $f$ by the Lagrange interpolation polynomial $L_{n}(f)$, say, of degree $\leqslant(n-1)$ and defined by

$$
L_{n}(f ; x)=\sum_{i=1}^{n} \frac{p_{n}(x) f\left(x_{i, n}\right)}{p_{n}^{\prime}\left(x_{i, n}\right)\left(x-x_{i, n}\right)}=\sum_{i=1}^{n} l_{i, n}(x) f\left(x_{i, n}\right),
$$

where $l_{i, n}\left(x_{j, n}\right)=\delta_{i, j}$ so that $f\left(x_{i, n}\right)=L_{n}\left(f ; x_{i, n}\right)$ for $i=1(1) n$. The quadrature sum $Q_{n}(f ; \lambda)$ is then defined by

$$
Q_{n}(f ; \lambda)=I\left(L_{n}(f) ; \lambda\right)=\sum_{i=1}^{n} A_{i, n}(\lambda) f\left(x_{i, n}\right),
$$

Received November 29, 1977.

AMS (MOS) subject classifications (1970). Primary 65D30; Secondary 41A10, 42A52. 
say. For $n=0,1,2, \ldots$, let $q_{n}$ denote the function defined by

$$
q_{n}(x)=f_{a}^{b} \frac{w(t) p_{n}(t)}{t-x} d t, \quad a<x<b
$$

Since we can write

$$
q_{n}(x)=\int_{a}^{b} w(t)\left(\frac{p_{n}(t)-p_{n}(x)}{t-x}\right) d t+p_{n}(x) q_{0}(x),
$$

we see that this set of functions exists provided $q_{0}$ exists, an assumption which is made throughout this paper. The coefficients $A_{i, n}(\lambda)$ in (1.4) are then given by

$$
A_{i, n}(\lambda)= \begin{cases}\frac{q_{n}\left(x_{i, n}\right)-q_{n}(\lambda)}{p_{n}^{\prime}\left(x_{i, n}\right)\left(x_{i, n}-\lambda\right)}, & \lambda \neq x_{i, n}, \\ q_{n}^{\prime}\left(x_{i, n}\right) / p_{n}^{\prime}\left(x_{i, n}\right), & \lambda=x_{i, n} .\end{cases}
$$

We now consider Hunter's method. He makes one variation to the method just described. Suppose first that $\lambda$ is not a zero of $p_{n}$, then Hunter approximates to $f$ by the Lagrange interpolation polynomial $L_{n+1}^{\dagger}(f)$, say, of degree $\leqslant n$ which is such that $L_{n+1}^{\dagger}\left(f ; x_{i, n}\right)=f\left(x_{i, n}\right), i=1(1) n$, and $L_{n+1}^{\dagger}(f ; \lambda)=f(\lambda)$. Thus, we find

$$
L_{n+1}^{\dagger}(f ; x)=\sum_{i=1}^{n} \frac{(x-\lambda) l_{i, n}(x)}{\left(x_{i, n}-\lambda\right)} f\left(x_{i, n}\right)+\frac{p_{n}(x)}{p_{n}(\lambda)} f(\lambda),
$$

provided $p_{n}(\lambda) \neq 0$. Hunter takes for his quadrature sum the $(n+1)$ point rule $Q_{n+1}^{\dagger}(f)$, say, where

$$
Q_{n+1}^{\dagger}(f ; \lambda)=I\left(L_{n+1}^{\dagger}(f) ; \lambda\right)=\sum_{i=1}^{n} \mu_{i, n} \frac{f\left(x_{i, n}\right)}{x_{i, n}-\lambda}+\frac{q_{n}(\lambda) f(\lambda)}{p_{n}(\lambda)},
$$

again provided that $p_{n}(\lambda) \neq 0$. The coefficients $\mu_{i, n}$ are the Christoffel numbers of the corresponding $n$-point Gaussian quadrature rule and are defined by

$$
\mu_{i, n}=\frac{q_{n}\left(x_{i, n}\right)}{p_{n}^{\prime}\left(x_{i, n}\right)}=\frac{k_{n} h_{n-1}}{k_{n-1} p_{n}^{\prime}\left(x_{i, n}\right) p_{n-1}\left(x_{i, n}\right)},
$$

for $i=1(1) n$, (see Szegö [20, Eq. (3.4.7)]).

If $\lambda$ is a zero of $p_{n}$, then appropriate modifications must be made to (1.7(a)) and (1.8(a)). Again, $L_{n+1}^{\dagger}(f)$ is chosen so that it takes the values of $f$ at the points $x_{i, n}$, $i=1(1) n$; but if $\lambda=x_{j, n}$, then we also require that $L_{n+1}^{\dagger \prime}\left(f ; x_{j, n}\right)=f^{\prime}\left(x_{j, n}\right)$. We find, when $\lambda=x_{j, n}$, that

$$
L_{n+1}^{\dagger}(f ; x)=\sum_{i=1 ; i \neq j}^{n} l_{i, n}(x)\left\{\frac{\left(x-x_{j, n}\right) f\left(x_{i, n}\right)-\left(x-x_{i, n}\right) f\left(x_{j, n}\right)}{\left(x_{i, n}-x_{j, n}\right)}\right\}
$$

$$
+l_{j, n}(x) f\left(x_{j, n}\right)+\frac{p_{n}(x)}{p_{n}^{\prime}\left(x_{j, n}\right)} f^{\prime}\left(x_{j, n}\right) .
$$

The quadrature sum is now given by 


$$
\begin{aligned}
Q_{n+1}^{\dagger}\left(f ; x_{j, n}\right)= & I\left(L_{n+1}^{\dagger}(f) ; x_{j, n}\right) \\
= & \sum_{i=1 ; i \neq j}^{n} \frac{q_{n}\left(x_{i, n}\right) f\left(x_{i, n}\right)-q_{n}\left(x_{j, n}\right) f\left(x_{j, n}\right)}{p_{n}^{\prime}\left(x_{i, n}\right)\left(x_{i, n}-x_{j, n}\right)} \\
& +\frac{q_{n}^{\prime}\left(x_{j, n}\right)}{p_{n}^{\prime}\left(x_{j, n}\right)} f\left(x_{j, n}\right)+\frac{q_{n}\left(x_{j, n}\right)}{p_{n}^{\prime}\left(x_{j, n}\right)} f^{\prime}\left(x_{j, n}\right) .
\end{aligned}
$$

It should be pointed out that particular cases of these two quadrature rules, or minor variants of them, have been given prior to 1972 by many authors, see [6], [8] , [12] , [15] , [17], [18] and [19]. Hunter [9] described his algorithm in the particular case of $w \equiv 1$ and the interval $(-1,1)$, so that the orthogonal polynomials were taken to be the Legendre polynomials $P_{n}$, although he did consider the case when the integrand possessed $m \geqslant 1$ simple poles on $(-1,1)$ and not just one simple pole at the point $\lambda$ as we have done here. Hunter's analysis has subsequently been generalized in various ways, see [3], [10] and [21]. Interpolation of $f$ at the points $\cos (\pi i / n), i=$ $0(1) n$, in the case when $w(x)=1$ has been proposed in [2] , [16] . A surprising omission from all the quoted references is that no one has given an expression for the remainder $I-Q$ in terms of higher derivatives of $f$. It is well known for Gaussian quadrature (see [5]), that if $g \in C^{2 n}(a, b)$, then

$$
\int_{a}^{b} w(x) g(x) d x=\sum_{i=1}^{n} \mu_{i, n} g\left(x_{i, n}\right)+h_{n} g^{(2 n)}(\eta) /\left[k_{n}^{2}(2 n) !\right]
$$

where $\eta \in(a, b)$, but no comparable result has been given for Gauss type quadrature rules for Cauchy principal value integrals. In Section 2 , we shall give such forms for the truncation errors for both the quadrature sums $Q_{n}(f ; \lambda)$ and $Q_{n+1}^{\dagger}(f ; \lambda)$.

The quadrature sum $Q_{n+1}^{\dagger}(f ; \lambda)$, as given by (1.8(a)), appears at first glance to be an attractive one since it can be considered as Gaussian quadrature applied to the integrand $f(x) /(x-\lambda)$, plus one extra term. However, it is apparent that if $\lambda$ is close to a zero of $p_{n}$, then $Q_{n+1}^{\dagger}(f ; \lambda)$ is likely to be obtained as the difference of two large numbers; if $\lambda$ is a zero of $p_{n}$, then we have already noted in (1.8(b)) the modifications that must be made to the quadrature sum. Furthermore, none of the authors who have written on this method have discussed the computation of $q_{n}(\lambda)$ for a given $\lambda$ and arbitrary $n$. In [14] the authors discussed an algorithm for the evaluation of $Q_{n}(f ; \lambda)$ based on the use of the Clenshaw recurrence algorithm [4]. In Section 3 we shall investigate how the method of [14] can be used for the evaluation of $Q_{n+1}^{\dagger}(f ; \lambda)$.

Finally, in Section 4 , we shall investigate the convergence of both $Q_{n}(f ; \lambda)$ and $Q_{n+1}^{\dagger}(f ; \lambda)$ to $I(f ; \lambda)$ as $n \rightarrow \infty$, when the interval $(a, b)$ is finite. Sufficient conditions will be given in each case to guarantee convergence. Although the convergence of $Q_{n}(f ; \lambda)$ to $I(f ; \lambda)$ has been discussed for the Jacobi weight function in [7], we shall first demonstrate convergence of $Q_{n}(f ; \lambda)$ to $I(f ; \lambda)$ for a more general weight function. The section concludes with a discussion of the convergence of $Q_{n+1}^{\dagger}(f ; \lambda)$ to $I(f ; \lambda)$, a problem which does not appear to have been discussed previously (but see $[22])$. 
2. Truncation Errors for $Q_{n}(f ; \lambda)$ and $Q_{n+1}^{\dagger}(f ; \lambda)$. We shall first consider the quadrature rule $Q_{n}(f ; \lambda)$, and let $R_{n}(f ; \lambda)=I(f ; \lambda)-Q_{n}(f ; \lambda)$ denote the truncation error at the point $\lambda \in(a, b)$. In order to obtain the required expression for $R_{n}(f ; \lambda)$, we shall assume that $f$ possesses a continuous derivative of order $(2 n+1)$ on $(a, b)$.

Let the function $s_{n}$ be defined on $(a, b)$ by

$$
s_{n}(x)=\frac{f(x)-L_{n}(f ; x)}{p_{n}(x)}=\sum_{i=1}^{n} \frac{f(x)-f\left(x_{i, n}\right)}{p_{n}^{\prime}\left(x_{i, n}\right)\left(x-x_{i, n}\right)},
$$

from (1.3), since $L_{n}(1 ; x) \equiv 1$. We observe that both $s_{n}$ and $s_{n}^{\prime}$ are defined even when $x$ is a zero of $p_{n}$, by taking the appropriate limits. Now, since $L_{n}(f)$ is the Lagrange interpolation polynomial to $f$ at the zeros of $p_{n}$, we also have (see [5]),

$$
f(x)-L_{n}(f ; x)=\left[p_{n}(x) f^{(n)}(\xi(x))\right] /\left[k_{n} n !\right],
$$

where $\xi \in(a, b)$ and also depends on $x$. Thus, in particular,

$$
s_{n}(\lambda)=f^{(n)}\left(\xi_{1}\right) /\left[k_{n} n !\right],
$$

say for some $\xi_{1} \in(a, b)$. From (1.4), (1.5) and (2.1) we can write

$$
\begin{aligned}
R_{n}(f ; \lambda) & =f_{a}^{b} \frac{w(x) p_{n}(x) s_{n}(x)}{x-\lambda} d x \\
& =s_{n}(\lambda) q_{n}(\lambda)+\int_{a}^{b} w(x) p_{n}(x)\left(\frac{s_{n}(x)-s_{n}(\lambda)}{x-\lambda}\right) d x \\
& =R_{n}^{1}(f ; \lambda)+R_{n}^{2}(f ; \lambda) \text { say }
\end{aligned}
$$

where, from (2.2),

$$
R_{n}^{1}(f ; \lambda)=s_{n}(\lambda) q_{n}(\lambda)=\left(q_{n}(\lambda) f^{(n)}\left(\xi_{1}\right)\right) /\left(k_{n} n !\right) .
$$

In order to obtain a similar expression for $R_{n}^{2}(f ; \lambda)$ we apply the $n$-point Gauss quadrature rule (1.10) to the integral. Since the integrand vanishes at each node point (even when $\lambda$ is a zero of $p_{n}$ ), we obtain

$$
R_{n}^{2}(f ; \lambda)=\frac{h_{n}}{k_{n}^{2}(2 n) !}\left\{\frac{d^{2 n}}{d x^{2 n}}\left[p_{n}(x)\left(\frac{s_{n}(x)-s_{n}(\lambda)}{x-\lambda}\right)\right]\right\}_{x=\eta},
$$

where $\eta \in(a, b)$. Let $P_{n-1}(x ; \lambda)$ denote the polynomial of degree $\leqslant(n-1)$ in $x$ defined by

$$
P_{n-1}(x ; \lambda)=\left[f(\lambda)-L_{n}(f ; x)-p_{n}(x) s_{n}(\lambda)\right] /(x-\lambda) .
$$

It is readily verified that the numerator is a polynomial of degree $\leqslant n$ in $x$ which is zero when $x=\lambda$. Then we can write

$$
p_{n}(x)\left(\frac{s_{n}(x)-s_{n}(\lambda)}{x-\lambda}\right)=P_{n-1}(x ; \lambda)+\int_{0}^{1} f^{\prime}(\lambda+(x-\lambda) \xi) d \xi,
$$

the integral being simply $(f(x)-f(\lambda)) /(x-\lambda)$. Substituting (2.6) into (2.5) gives

$$
R_{n}^{2}(f ; \lambda)=\frac{h_{n}}{k_{n}^{2}(2 n) !}\left\{\frac{d^{2 n}}{d x^{2 n}}\left[\int_{0}^{1} f^{\prime}(\lambda+(x-\lambda) \xi) d \xi\right]\right\}_{x=\eta}
$$


On inverting the order of differentiation and integration, and applying the mean value theorem to the resulting integral, we find

$$
R_{n}^{2}(f ; \lambda)=\left(h_{n} f^{(2 n+1)}\left(\xi_{2}\right)\right) /\left(k_{n}^{2}(2 n+1) !\right),
$$

where $\xi_{2} \in(a, b)$. Combining (2.4) and (2.8) gives us our first required result, that is

$$
R_{n}(f ; \lambda)=\frac{q_{n}(\lambda)}{k_{n} n !} f^{(n)}\left(\xi_{1}\right)+\frac{h_{n}}{k_{n}^{2}(2 n+1) !} f^{(2 n+1)}\left(\xi_{2}\right),
$$

where $\xi_{1}, \xi_{2} \in(a, b)$ and depend upon $\lambda$.

The truncation error $R_{n+1}^{\dagger}(f ; \lambda)=I(f ; \lambda)-Q_{n+1}^{\dagger}(f ; \lambda)$ for the quadrature sum $Q_{n+1}^{\dagger}(f ; \lambda)$ may now be readily obtained. Let us suppose first that $\lambda$ is not a zero of $p_{n}$. From (2.3) we can write

$$
I(f ; \lambda)=\sum_{i=1}^{n} A_{i, n}(\lambda) f\left(x_{i, n}\right)+s_{n}(\lambda) q_{n}(\lambda)+R_{n}^{2}(f ; \lambda) .
$$

On substituting for $s_{n}(\lambda)$ from (2.1), recalling the definition of $A_{i, n}(\lambda)$ from (1.6), and using the definition of $Q_{n+1}^{\dagger}(f ; \lambda)$ from (1.8(a)), we find from (2.8) that

$$
I(f ; \lambda)=Q_{n+1}^{\dagger}(f ; \lambda)+\frac{h_{n}}{k_{n}^{2}(2 n+1) !} f^{(2 n+1)}\left(\xi_{2}\right),
$$

where $\xi_{2} \in(a, b)$. If $\lambda$ is a zero of $p_{n}$, then it can be verified, on using (1.8(b)), that (2.11) is again valid, thus completing our analysis.

We see from (2.9) and (2.11), respectively, that $Q_{n}(f ; \lambda)$ is exact whenever $f$ is a polynomial of degree $\leqslant(n-1)$ and $Q_{n+1}^{\dagger}(f ; \lambda)$ is exact whenever $f$ is a polynomial of degree $\leqslant 2 n$. This follows from the construction of the quadrature rules and has been observed previously. From (2.10) we see that the quadrature rules are the same if $\lambda$ is chosen so that it is a zero of $q_{n}$.

3. An Algorithm for Evaluating $Q_{n+1}^{\dagger}(f ; \lambda)$. We shall first outline the algorithm described in [14], for the evaluation of the quadrature sum $Q_{n}(f ; \lambda)$. This depended upon the observation that both $p_{k}$ and $q_{k}$ satisfy the same linear three term recurrence relation, which we shall suppose is given by

$$
u_{k+1}(x)=\left(A_{k} x+B_{k}\right) u_{k}(x)-C_{k} u_{k-1}(x), \quad A_{k}=k_{k+1} / k_{k},
$$

for $k=0,1,2, \ldots$. In [14], this recurrence relation was used to set up a codiagonal matrix $M_{n}$, say, of order $n$, whose eigenvalues are the zeros $x_{i, n}, i=1(1) n$, of the polynomial $p_{n}$. To each $x_{i, n}$ the corresponding eigenvector $\mathbf{p}_{i}$ is given by $\mathbf{p}_{i}^{T}=$ $\left(p_{0}\left(x_{i, n}\right), p_{1}\left(x_{i, n}\right), \ldots, p_{n-1}\left(x_{i, n}\right)\right)$ from which the Christoffel numbers $\mu_{i, n}$ may be computed since $\mu_{i, n}=\left(\sum_{j=0}^{n-1} p_{j}^{2}\left(x_{i, n}\right)\right)^{-1}$, for $i=1(1) n$. If the Lagrange interpolation polynomial $L_{n}(f ; x)$ is written as

$$
L_{n}(f ; x)=\sum_{k=0}^{n-1} a_{k, n} p_{k}(x)
$$

where

$$
a_{k, n}=h_{k}^{-1} \int_{a}^{b} w(x) L_{n}(f ; x) p_{k}(x) d x=h_{k}^{-1} \sum_{i=1}^{n} \mu_{i, n} p_{k}\left(x_{i, n}\right) f\left(x_{i, n}\right)
$$


for $k=O(1)(n-1)$, then the quadrature sum $Q_{n}(f ; \lambda)$ is given by

$$
Q_{n}(f ; \lambda)=\sum_{k=0}^{n-1} a_{k, n} q_{k}(\lambda)
$$

Since the functions $q_{k}$ satisfy the recurrence relation (3.1), Clenshaw's algorithm [4] may be used to evaluate the sum in (3.4), for a given value of $\lambda$. If we put $q_{-1}(\lambda)$ $\equiv 0$, then all that is required to implement this algorithm is an explicit expression for $q_{0}(\lambda)$.

We now consider a similar algorithm for the evaluation of $Q_{n+1}^{\dagger}(f ; \lambda)$. If we write

$$
L_{n+1}^{\dagger}(f ; x)=\sum_{k=0}^{n} a_{k, n+1}^{\dagger} p_{k}(x)
$$

where

$$
a_{k, n+1}^{\dagger}=h_{k}^{-1} \int_{a}^{b} w(x) L_{n+1}^{\dagger}(f ; x) p_{k}(x) d x,
$$

then we find, on using (1.10) and the orthogonality of the $\left\{p_{k}\right\}$, that

$$
a_{k, n+1}^{\dagger}=a_{k, n}, \text { for } k=0(1)(n-1) \text {. }
$$

Suppose first that $\lambda$ is not a zero of $p_{n}$. Since

$$
h_{n}^{-1} \int_{a}^{b} w(x)\left[(x-\lambda) /\left(x-x_{i, n}\right)\right] p_{n}^{2}(x) d x=1, \text { for } i=1(1) n,
$$

it follows from (3.6) and (1.7(a)) that

$$
a_{n, n+1}^{\dagger}=\sum_{i=1}^{n} \frac{1}{p_{n}^{\prime}\left(x_{i, n}\right)}\left(\frac{f(\lambda)-f\left(x_{i, n}\right)}{\lambda-x_{i, n}}\right) \text {. }
$$

On the other hand, if $\lambda$ is a zero $x_{j, n}$ of $p_{n}$, then again, from (3.6) and (1.7(b)), we find that

$$
a_{n, n+1}^{\dagger}=\sum_{i=1 ; i \neq j}^{n} \frac{1}{p_{n}^{\prime}\left(x_{i, n}\right)}\left(\frac{f\left(x_{j, n}\right)-f\left(x_{i, n}\right)}{x_{j, n}-x_{i, n}}\right)+\frac{f^{\prime}\left(x_{j, n}\right)}{p_{n}^{\prime}\left(x_{j, n}\right)} .
$$

The values of $1 / p_{n}^{\prime}\left(x_{i, n}\right), i=1(1) n$, may be readily obtained from the eigenvectors $\mathbf{p}_{i}$ of the matrix $M_{n}$, since we have $1 / p_{n}^{\prime}\left(x_{i, n}\right)=\mu_{i, n} p_{n-1}\left(x_{i, n}\right) /\left(A_{n-1} h_{n-1}\right)$. Thus

$$
Q_{n+1}^{\dagger}(f ; \lambda)=\sum_{k=0}^{n} \mathrm{a}_{k, n+1}^{\dagger} q_{k}(\lambda)
$$

which again may be summed by means of the Clenshaw algorithm. Compared with the evaluation of $Q_{n}(f ; \lambda)$, the quadrature sum $Q_{n+1}^{\dagger}(f ; \lambda)$ requires the computation of one extra coefficient $a_{n, n+1}^{\dagger}$. Once a procedure has been provided for the evaluation of $\left(f(\lambda)-f\left(x_{i, n}\right)\right) /\left(\lambda-x_{i, n}\right)$, particularly when $\lambda$ is either close to, or equal to, a zero of $p_{n}$, then we have an algorithm for the evaluation of $Q_{n+1}^{\dagger}(f ; \lambda)$ which will be comparable to that for $Q_{n}(f ; \lambda)$.

4. Convergence Theorems for $Q_{n}$ and $Q_{n+1}^{\dagger}$. Throughout this section we assume that the interval $(a, b)$ is finite and, without any loss of generality, we shall take it to be $(-1,1)$.

Firstly, we shall consider the convergence of $Q_{n}(f, \lambda)$ to $I(f ; \lambda)$ for a given 
$\lambda \in(-1,1)$. In order to do this we need to make an assumption concerning the behavior of the functions $q_{k}(\lambda)$. We assume that

$$
\left|q_{k}(\lambda)\right| \leqslant A(\lambda) h_{k}^{1 / 2}, \quad k=0,1,2, \ldots,
$$

where $A$ depends upon $\lambda$, but is independent of $k$. This assumption does not appear to be very restrictive. It is readily verified that it is satisfied by the Chebyshev polynomials of both kinds, and the Legendre polynomials. Furthermore, following an analysis given by Barrett [1], Paget [13] has obtained the asymptotic form of $q_{k}(\lambda)$ for large $k$ under the fairly general assumption that both $w$ and $\log w$ are integrable on $(-1,1)$. From this asymptotic analysis, it can be shown that (4.1) is satisfied for the resulting $q_{k}$.

A function $f$ is said to be Hölder continuous of order $\mu$ on $[-1,1]$ if $\mid f\left(x_{1}\right)-$ $f\left(x_{2}\right)|\leqslant A| x_{1}-\left.x_{2}\right|^{\mu}$ for any pair of points $x_{1}, x_{2} \in[-1,1]$, where $A$ is independent of $x_{1}$ and $x_{2}$.

THEOREM 4.1. Suppose $w$ is such that $q_{k}$ exist for $k=0,1,2, \ldots$ and furthermore that (4.1) is satisfied. If $f$ is Hölder continuous of order $\mu$ on $[-1,1]$ with $1 / 2<$ $\mu \leqslant 1$, then for a given $\lambda \in(-1,1)$,

$$
\lim _{n \rightarrow \infty} Q_{n}(f ; \lambda)=I(f ; \lambda) \text {. }
$$

Proof. Let $p_{n-1}^{*}$ denote the polynomial, of degree $\leqslant(n-1)$, of best uniform approximation to $f$ on $[-1,1]$. Write $r_{n-1}^{*}(x)=f(x)-p_{n-1}^{*}(x)$, and

$$
E_{n-1}(f)=\max _{x \in[-1,1]}\left|r_{n-1}^{*}(x)\right| \text {. }
$$

It is well known that $E_{n-1}(f) \leqslant C n^{-\mu}$, but Kalandiya [11] has further shown that $r_{n-1}^{*}$ is Hölder continuous of order $\nu$ say where $0<\nu<\mu / 2$. Furthermore, for any $x_{1}, x_{2} \in[-1,1]$,

$$
\left|r_{n-1}^{*}\left(x_{1}\right)-r_{n-1}^{*}\left(x_{2}\right)\right| \leqslant B(\nu)(n-1)^{-(\mu-2 \nu)}\left|x_{1}-x_{2}\right|^{\nu}
$$

where $B(\nu)$ is independent of $n, x_{1}$ and $x_{2}$. Since

$$
\begin{aligned}
R_{n}(f ; \lambda)= & \int_{-1}^{1} w(x)\left(\frac{r_{n-1}^{*}(x)-r_{n-1}^{*}(\lambda)}{x-\lambda}\right) d x+r_{n-1}^{*}(\lambda) q_{0}(\lambda) / k_{0} \\
& -f_{-1}^{1} \frac{w(x) L_{n}\left(r_{n-1}^{*} ; x\right)}{x-\lambda} d x
\end{aligned}
$$

then from (4.3), the first integral on the right of (4.4) exists and tends to zero in the limit as $n \rightarrow \infty$. The second term on the right of (4.4) also tends to zero as $n \rightarrow \infty$, so that the convergence of $R_{n}$ to zero will depend on

$$
J_{n}(\lambda)=f_{-1}^{1}\left[w(x) L_{n}\left(r_{n-1}^{*} ; x\right) /(x-\lambda)\right] d x
$$

Now $L_{n}\left(r_{n-1}^{*} ; x\right)$ is a polynomial of degree $\leqslant(n-1)$; if we write

$$
L_{n}\left(r_{n-1}^{*} ; x\right)=\sum_{k=0}^{n-1} \rho_{k} p_{k}(x),
$$

then we shall have

$$
J_{n}(\lambda)=\sum_{k=0}^{n-1}\left(h_{k}^{1 / 2} \rho_{k}\right)\left(h_{k}^{-1 / 2} q_{k}(\lambda)\right)
$$


By Hölder's inequality,

$$
\left|J_{n}(\lambda)\right| \leqslant\left(\sum_{k=0}^{n-1} h_{k} \rho_{k}^{2}\right)^{1 / 2}\left(\sum_{k=0}^{n-1} h_{k}^{-1} q_{k}^{2}(\lambda)\right)^{1 / 2}
$$

since

$$
\int_{-1}^{1} w(x) L_{n}^{2}\left(r_{n-1}^{*} ; x\right) d x=\sum_{k=0}^{n-1} h_{k} \rho_{k}^{2},
$$

and using (4.1), we obtain

$$
\left|J_{n}(\lambda)\right| \leqslant\left(\int_{-1}^{1} w(x) L_{n}^{2}\left(r_{n-1}^{*} ; x\right) d x\right)^{1 / 2} A(\lambda) n^{1 / 2} .
$$

On evaluating the integral using (1.10), recalling that

$$
L_{n}\left(r_{n-1}^{*} ; x\right)=\sum_{i=1}^{n} l_{i, n}(x) r_{n-1}^{*}\left(x_{i, n}\right),
$$

noting that $\left|r_{n-1}^{*}\left(x_{i, n}\right)\right| \leqslant E_{n-1}(f)$, for $i=1(1) n$, and $\Sigma_{k=0}^{n} \mu_{k, n}=\int_{-1}^{1} w(x) d x$, we find

$$
\left|J_{n}(\lambda)\right| \leqslant A(\lambda)\left(\int_{-1}^{1} w(x) d x\right)^{1 / 2} n^{1 / 2} E_{n-1}(f) .
$$

Since $E_{n-1}(f) \leqslant C n^{-\mu}$, where $C$ is some constant, and $\mu>1 / 2$ we have $\lim _{n \rightarrow \infty} J_{n}(\lambda)$ $=0$, and the theorem is proved.

It is worth noting that convergence of $Q_{n}(f ; \lambda)$ to $I(f ; \lambda)$ has previously been discussed by Elliott and Paget [7] in the particular case when $w(x)=(1-x)^{\alpha}(1+x)^{\beta}$, $\alpha, \beta>-1$. They found, using an asymptotic analysis, that there was convergence for all Hölder continuous $f$ such that $0<\mu \leqslant 1$. Since $L_{n}\left(r_{n-1}^{*} ; x\right)$ is a polynomial of degree $\leqslant(n-1)$, one can write

$$
J_{n}(\lambda)=\sum_{i=1}^{n} A_{i, n}(\lambda) r_{n-1}^{*}\left(x_{i, n}\right)
$$

so that $\left|J_{n}(\lambda)\right| \leqslant E_{n-1}(f) \sum_{i=1}^{n}\left|A_{i, n}(\lambda)\right|$. In [7], the authors showed that for large $n$, $\sum_{i=1}^{n}\left|A_{i, n}(\lambda)\right| \leqslant K+L \log n$, where $K, L$ are independent of $n$. We have not attempted an asymptotic analysis in this more general case.

We shall now consider convergence of the sequence $\left\{Q_{n}^{\dagger}(f ; \lambda)\right\}$ to $I(f ; \lambda)$ for a given $\lambda \in(-1,1)$ as $n \rightarrow \infty$. From the construction of the rules $Q_{n}^{\dagger}(f ; \lambda)$ it is obvious that we need to assume differentiability of $f$.

THEOREM 4.2. Suppose $f^{\prime}$ is continuous on $[-1,1]$, then for any given $\lambda \in$ $(-1,1)$,

$$
\lim _{n \rightarrow \infty} Q_{n}^{\dagger}(f ; \lambda)=I(f ; \lambda) .
$$

Proof. Let $g(x, \lambda)$ be defined by

$$
g(x, \lambda)=\left\{\begin{array}{l}
(f(x)-f(\lambda)) /(x-\lambda), \quad x \neq \lambda, \\
f^{\prime}(\lambda), \quad x=\lambda .
\end{array}\right.
$$

From (1.1), (1.8(a)), (1.8(b)) it can be verified that if we write $R_{n+1}^{\dagger}(f ; \lambda)=I(f ; \lambda)$ $-Q_{n+1}^{\dagger}(f ; \lambda)$, then

$$
R_{n+1}^{\dagger}(f ; \lambda)=\int_{-1}^{1} w(x) g(x, \lambda) d x-\sum_{k=1}^{n} \mu_{k, n} g\left(x_{k, n}, \lambda\right)+f(\lambda) R_{n+1}^{\dagger}(1 ; \lambda)
$$


this result being valid irrespective of whether $\lambda$ is or is not a zero of $p_{n}$. From (2.11), we have trivially that $R_{n+1}^{\dagger}(1 ; \lambda)=0$. Thus, $R_{n+1}^{\dagger}(f ; \lambda)$ is given by the remainder when $n$-point Gauss quadrature (based on the zeros of $p_{n}$ ), is applied to the function $g$. Since under the stated conditions on $f$, the function $g$ is continuous it follows (see, for example, [5]) that $\lim _{n \rightarrow \infty} R_{n+1}^{\dagger}(f ; \lambda)=0$, as required.

Mathematics Department

University of Tasmania

Hobart, Tasmania, Australia

1. W. BARRETT, "An asymptotic formula relating to orthogonal polynomials," J. London Math. Soc. (2), v. 6, 1973, pp. 701-704. MR 48 \#593.

2. M. M. CHAWLA \& N. JAYARAJAN, "Quadrature formulas for Cauchy principal value integrals," Computing, v. 15, 1975, pp. 347-355. MR 54 \#4068.

3. M. M. CHAWLA \& T. R. RAMAKRISHNAN, "Modified Gauss-Jacobi quadrature formulas for the numerical evaluation of Cauchy type singular integrals," BIT, v. 14, 1974, pp. 14-21. MR 51 \#9437.

4. C. W. CLENSHAW, "A note on the summation of Chebyshev series," Math. Comp., v. 9, 1955, pp. 118-120. MR 17, 194.

5. P. J. DAVIS \& P. RABINOWITZ, Methods of Numerical Integration, Academic Press, New York, 1975.

6. L. M. DELVES, "The numerical evaluation of principal value integrals," Comput. J., v. 10, 1967/1968, pp. 389-391. MR $36 \# 4802$.

7. D. ELLIOTT \& D. F. PAGET, "On the convergence of a quadrature rule for evaluating certain Cauchy principal value integrals," Numer. Math., v. 23, 1975, pp. 311-319; v. 25, 1976, pp. 287-289. MR 52\#1115;53\#14870.

8. F. ERDOGAN \& G. D. GUPTA, "On the numerical solution of singular integral equations," Quart. Appl. Math., v. 29, 1972, pp. 525-534. MR 53 \#12042.

9. D. B. HUNTER, "Some Gauss-type formulae for the evaluation of Cauchy principal values of integrals," Numer. Math., v. 19, 1972, pp. 419-424. MR 47 \#7899.

10. N. I. IOAKIMIDIS \& P. S. THEOCARIS, "The Gauss-Hermite numerical integration method for the solution of the plane elastic problem of semi-infinite periodic cracks," Internat. J. Engrg. Sci., v. 15, 1977, pp. $271-280$.

11. A. I. KALANDIYA, "On a direct method of solution of an equation in wing theory with an application to the theory of elasticity," Mat. Sb., v. 42, 1957, pp. 249-272. (Russian) MR 21 \#6800.

12. V. I. LEBEDEV \& O. V. BABURIN, "Calculation of the principal values, weights and nodes of the Gauss quadrature formulae of integrals," U.S.S.R. Comput. Math. and Math. Phys., v. 5, 1965, pp. 81-92. MR $32 \# 6670$.

13. D. F. PAGET, Generalized Product Integration, Ph. D. thesis, Univ. of Tasmania, 1976.

14. D. F. PAGET \& D. ELLIOTT, "An algorithm for the numerical evaluation of certain Cauchy principal value integrals," Numer. Math., v. 19, 1972, pp. 373-385. MR 51 \#2256.

15. R. PIESSENS, "Numerical evaluation of Cauchy principal values of integrals," $B I T, v$. 10, 1970, pp. 476-480. MR 43 \#7066.

16. R. PIESSENS, M. VAN ROY-BRANDERS \& I. MERTENS, "The automatic evaluation of Cauchy principal value integrals," Angewandte Informatik, v. 1, 1976, pp. 31-35.

17. J. F. PRICE, Discussion of Quadrature Formulas for Use on Digital Computers, Rep. D1-82-0052, Boeing Sci. Res. Labs., 1960.

18. G. N. PYKHTEEV, "On the evaluation of certain singular integrals with a kernel of the Cauchy type," J. Appl. Math. Mech., v. 23, 1959, pp. 1536-1548. MR 22 \#12698.

19. V. J.E. STARK, "A generalized quadrature formula for Cauchy integrals," AIAA J., v. 9, 1971 , pp. 1854-1855. MR 45 \#4639.

20. G. SZEGÖ, Orthogonal Polynomials, 3rd ed., Amer. Math. Soc. Colloq. Publ., v. 23, Amer. Math. Soc., Providence, R. I., 1967. MR 1, 14.

21. P. S. THEOCARIS \& N. I. IOAKIMIDIS, "Numerical solution of Cauchy type singular integral equations," Trans. Acad. Athens, v. 40, 1977, pp. 1-39.

22. G. J. TSAMASPHYROS \& P. S. THEOCARIS, "On the convergence of a Gauss quadrature rule for evaluation of Cauchy type singular integrals," BIT, v. 17, 1977, pp. 458-464. 